\title{
Parenting styles, family structure and adolescent dietary behaviour
}

\author{
Natalie Pearson* , Andrew J Atkin, Stuart JH Biddle, Trish Gorely and \\ Charlotte Edwardson \\ School of Sport, Exercise \& Health Sciences, Loughborough University, Loughborough, Leicestershire, \\ LE 11 3TU, UK
}

Submitted 15 May 2009: Accepted 20 October 2009: First published online 3 December 2009

\begin{abstract}
Objective: To examine associations between parenting styles, family structure and aspects of adolescent dietary behaviour.

Design: Cross-sectional study.

Setting: Secondary schools in the East Midlands, UK.

Subjects: Adolescents aged 12-16 years ( $n$ 328, 57\% boys) completed an FFQ assessing their consumption of fruit, vegetables, unhealthy snacks and breakfast. Adolescents provided information on parental and sibling status and completed a seventeen-item instrument measuring the general parenting style dimensions of involvement and strictness, from which four styles were derived: indulgent, neglectful, authoritarian, authoritative.

Results: After controlling for adolescent gender and age, analysis of covariance revealed no significant interactions between parenting style and family structure variables for any of the dietary behaviours assessed. Significant main effects for family structure were observed only for breakfast consumption, with adolescents from dual-parent families $(P<0 \cdot 01)$ and those with no brothers $(P<0 \cdot 05)$ eating breakfast on more days per week than those from single-parent families and those with one or more brother, respectively. Significant main effects for parenting style were observed for all dietary behaviours apart from vegetable consumption. Adolescents who described their parents as authoritative ate more fruit per day, fewer unhealthy snacks per day, and ate breakfast on more days per week than those who described their parents as neglectful.

Conclusions: The positive associations between authoritative parenting style and adolescent dietary behaviour transcend family structure. Future research should be food-specific and assess the efficacy of strategies promoting the central attributes of an authoritative parenting style on the dietary behaviours of adolescents from a variety of family structures.
\end{abstract}

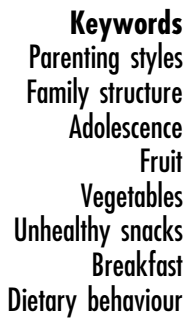

Dietary behaviour is a major determinant of chronic disease and the promotion of healthful dietary behaviours in all populations, including adolescents, is a public health priority. It is widely acknowledged that adolescents eat fewer fruits and vegetables than is recommended for health $^{(1,2)}$, and studies have shown that many adolescents engage in health-compromising behaviours such as frequent dieting, meal skipping and consumption of foods high in fats, sodium and sugar ${ }^{(3)}$. Diets rich in fruit and vegetables have important short- and long-term healthprotective effects ${ }^{(4,5)}$, whereas diets including high intakes of unhealthy snacks, and frequent breakfast skipping, have been associated with detriments to health, such as higher BMI ${ }^{(6,7)}$. Dietary behaviours established in adolescence are more likely to persist into adulthood ${ }^{(8,9)}$. Examining the influences on adolescent dietary behaviours is integral for the development of targeted intervention strategies.

The family environment has been identified as a critical context for the development of eating behaviours ${ }^{(10)}$. Research suggests that within the family environment the quality of the parent-adolescent relationship has a significant impact on the development of adolescent healthrisk behaviours ${ }^{(11)}$. Such research has found parental warmth and involvement, emotional support, appropriate granting of autonomy, and clear, bidirectional communication to be positively associated with more healthful behaviour in children and adolescents ${ }^{(12,13)}$. This clustering of parental attributes is known as 'authoritative' parenting, one of several styles of parenting identified by Baumrind ${ }^{(14,15)}$. Parenting style is a global concept regarded as the general emotional climate for parent-adolescent interactions 
across a wide range of situations and is classified according to two dimensions of parental behaviour - 'strictness', or parental control, and 'involvement', or parental warmth and acceptance ${ }^{(13)}$ - resulting in four parenting styles: authoritative (parents who are strict but also involved), authoritarian (parents who are strict but uninvolved), indulgent (parents who are involved, but not strict) and neglectful (parents who are neither strict nor involved). Within these general parenting styles, parents also display more specific parenting practices, which are typically context-specific behaviours ${ }^{(16)}$ (e.g. rules about the consumption of certain foods).

Research examining the influence of food-related parenting practices has yielded conflicting results. Several studies show that strict parenting practices may have adverse effects, such as increasing preference for and intake of the restricted foods ${ }^{(17,18)}$, whereas other studies suggest that adolescents have a healthier diet when they report more food-related rules in their family ${ }^{(19,20)}$. Such conflicting results suggest that the influence of parenting practices may differ depending on the general style of parenting. In fact, Darling and Steinberg ${ }^{(16)}$ have argued that the effectiveness of specific parenting practices is moderated by the general parenting style. The influence of parenting style on adolescent dietary behaviours has not been comprehensively explored, yet such characteristics could affect adolescent dietary behaviours by influencing the context in which adolescents experience certain foods. For example, encouraging adolescents to eat a healthy breakfast may be successful for parents who combine strictness and involvement in the interactions with their child (i.e. authoritative parenting style) and unsuccessful for parents with a different parenting style. Understanding the influence of certain parenting styles on adolescent dietary behaviours will allow for a greater understanding of the emotional climates within which positive and negative health behaviours are borne.

The current literature examining associations between parenting styles and adolescent dietary behaviour is limited to fruit and vegetable consumption and has shown equivocal results. Three studies found no association between parenting style and fruit and vegetable consumption among children and adolescents ${ }^{(21-23)}$, while some evidence has shown a positive association between an authoritative parenting style and fruit and vegetable consumption in adolescents ${ }^{(24,25)}$. While these studies tell us whether positive parent-adolescent interactions contribute to adolescents' development of fruit and vegetable eating behaviours, they do not reveal whether the parental processes that are important in one family context (e.g. dual-parent families) are equally so in others (e.g. single-parent families). In light of the changed and changing demography of youth, research that takes into account the wider context within which parent-adolescent interactions occur is imperative. There is good reason to believe that the effects of specific parenting styles on adolescents' eating behaviours may be moderated by the larger context in which adolescents live ${ }^{(26)}$.

Each parenting style reflects different patterns in parental behaviours, values and practice, which are, in turn, influenced by aspects of the wider family environment in which parents and adolescents live (e.g. marital status, sibling status). Research has shown that marital relationships exert considerable influence on parenting processes and behaviours, and that adolescents from single-parent homes are likely to have more responsibility, independence and decision-making power than those in dual-parent families ${ }^{(27)}$. Similarly, the number of children in a family, and the gender of siblings, influence parent-adolescent interactions ${ }^{(28)}$. Adolescent dietary behaviours are also known to vary as a function of the family structure in which they live. For example, research has shown that adolescents from dual-parent families are more likely to regularly eat breakfast $^{(29)}$ and are less likely to increase their consumption of unhealthy snacks over a 2 -year period ${ }^{(30)}$ compared with those in single-parent families.

The aim of the present study is to build on the limited body of research examining associations between parenting styles, family structure and adolescent dietary behaviour. Parenting is a learned process and the identification of parenting styles that are associated with positive dietary behaviours in adolescents across various family structures is fundamental for identifying target groups and for developing tailored intervention strategies to promote healthy eating. This may help identify potential moderators of intervention effectiveness. Therefore the present study aims to examine cross-sectional associations between parenting styles, family structure and adolescent consumption of fruit, vegetables, unhealthy snacks and breakfast.

\section{Methods}

\section{Sample and procedure}

Cross-sectional data were collected between October 2007 and June 2008. Study procedures were approved by the Ethical Advisory Committee of the host university. Data were obtained from adolescents (12-16 years) recruited from three secondary schools in the East Midlands region of the UK. Staff at participating schools selected a subset of their classes for participation. All students from nominated classes ( $n$ 363) were eligible and received written information on the project. Consent was sought from parents prior to the study and adolescent participants provided assent before completing written surveys during class. In total, 328 pupils provided consent and completed the survey ( $90 \%$ response rate).

\section{Measures}

\section{Family structure}

Participants completed questionnaires during Physical Education or Personal, Social and Health Education lessons, 
under the supervision of trained researchers and class teachers. Specific characteristics of family structure, including whom the adolescents lived with at home and the number of brothers and sisters, were assessed. Parental status was dichotomized as dual-parent (i.e. lived with mother and father or de facto) or single-parent (i.e. lived with only mother or only father). Sibling status was categorised into 'none' or 'one or more' siblings, 'none' or 'one or more' brothers and 'none' or 'one or more' sisters. Demographic information, including date of birth and ethnicity, was provided by the school. Socio-economic position was determined using the Index of Multiple Deprivation, a measure of compound social and material deprivation, calculated from a variety of data including income, employment, health, education and housing. It is based on the postcode of the participant's home, and thus represents an area-level approximation of socio-economic position.

\section{Parenting styles}

Adolescents completed a seventeen-item instrument, based on previous work ${ }^{(20,24,31,32)}$, measuring the general parenting style dimensions of involvement and strictness ${ }^{(33)}$. The involvement scale was measured by ten items (Cronbach's $\alpha=0 \cdot 85$ ), for example: 'When I have a bad result at school, my parents encourage me to do better'. Strictness was measured by seven items (Cronbach's $\alpha=0 \cdot 73$ ), for example: 'My parents know exactly where I am after school'. Response categories for both scales were 'strongly disagree', 'disagree', 'neither agree nor disagree', 'agree' and 'strongly agree'. Based on the median value of both scales, four parenting categories were defined. Authoritative parents ( $n$ 124) were those who scored in the upper half on both involvement and strictness, whereas neglectful parents ( $n$ 91) scored in the lower half on both dimensions. Authoritarian parents ( $n$ 59) scored in the lower half on involvement and in the upper half on strictness. Indulgent parents ( $n 53)$ scored in the upper half on involvement and in the lower half on strictness.

\section{Adolescent dietary behaviour}

Adolescent food intake was assessed using a thirty-item FFQ, based on the previously validated Youth/Adolescent Food Frequency Questionnaire (YAQ) ${ }^{(34)}$. Adolescents were asked how often they ate ten different fruits, twelve different vegetables, and eight different unhealthy snacks or types of unhealthy snacks (e.g. crisps, chocolate) in the past month. Responses to questions on the frequency of consumption of specific fruits, vegetables and snacks were summed to assess average total daily consumption. Breakfast consumption was assessed with a single item asking adolescents how often they ate breakfast in the past 7 days.

\section{Statistical analyses}

All analyses were conducted using the SPSS statistical software package version 16.0 (SPSS Inc., Chicago, IL,
USA). Descriptive statistics were used to summarise the sociodemographic and dietary characteristics of the sample. Pearson's $\chi^{2}$ tests were used to examine gender and age group differences in parenting style and family structure variables. Independent $t$ tests were performed to examine gender and age group differences in mean fruit, vegetable and snack consumption per day and mean breakfast consumption per week. As there were some significant differences in behaviours by gender and age group, gender and age were entered as covariates in all further analyses.

Two-way analysis of covariance (ANCOVA) was performed to examine whether fruit consumption, vegetable consumption, unhealthy snack consumption and breakfast consumption differed between the four parenting styles and between the family structure variables. For each analysis, the main effect of parenting style, the main effect of family structure and the interaction between parenting style and family structure are reported in the tables. Post hoc tests were used to detail specific differences between the four types of parenting style for each of the outcome variables. A significance level of 0.05 was applied for all analyses.

\section{Results}

\section{Sample characteristics}

Most of the sample was white British (97\%) and upper socio-economic position (67\%). Over half of the sample were boys (57\%) and younger adolescents (52\%). The mean age of the younger adolescents was 13.3 years and the mean age of the older adolescents was $15 \cdot 6$ years. Table 1 presents the distribution of parenting styles and family structure variables by gender and age group. A higher proportion of boys, compared with girls, had one or more sister $(P<0 \cdot 01)$. A higher proportion of older adolescents, compared with younger adolescents, described their parents as neglectful and a higher proportion of younger adolescents, compared with older adolescents, described their parents as authoritative $(P<0 \cdot 001)$.

\section{Adolescent dietary bebaviours}

Significant differences in dietary behaviours were found between genders (Table 2). Compared with girls, boys reported eating more snacks per day and reported eating breakfast on more days per week $(P<0 \cdot 01)$. Dietary behaviours were also associated with age group. Compared with younger adolescents, older adolescents reported eating more vegetables per day $(P<0 \cdot 01)$.

\section{Relationship between parenting styles, family structure and adolescent dietary behaviours}

After controlling for gender and age of the adolescent, ANCOVA revealed no significant interactions between parenting style and family structure variables for any of 
Table 1 Frequency of parenting styles and family structure variables according to gender and age group of adolescents, East Midlands, UK, October 2007 to June 2008

\begin{tabular}{|c|c|c|c|c|c|}
\hline & \multirow[b]{2}{*}{ Total sample (\%) (n 328) } & \multicolumn{2}{|c|}{ Gender (\%) } & \multicolumn{2}{|c|}{ Age group (\%) } \\
\hline & & Boys ( $n$ 186) & Girls (n 142) & Younger adolescents ( $n$ 170) & Older adolescents ( $n$ 158) \\
\hline \multicolumn{6}{|l|}{ Parenting style } \\
\hline Indulgent & $16 \cdot 2$ & $18 \cdot 8$ & $12 \cdot 7$ & $10 \cdot 0$ & $22 \cdot 8$ \\
\hline Neglectful & $27 \cdot 7$ & $30 \cdot 7$ & $23 \cdot 9$ & $20 \cdot 0$ & $36 \cdot 1^{\star \star \star}$ \\
\hline Authoritarian & $17 \cdot 9$ & $15 \cdot 6$ & $21 \cdot 1$ & $20 \cdot 6$ & $15 \cdot 2$ \\
\hline Authoritative & $38 \cdot 2$ & $34 \cdot 9$ & $42 \cdot 3$ & $49 \cdot 4$ & $25 \cdot 9^{\star \star \star}$ \\
\hline \multicolumn{6}{|l|}{ Parental status } \\
\hline Single parent & $15 \cdot 5$ & $15 \cdot 1$ & $16 \cdot 2$ & $16 \cdot 5$ & $14 \cdot 6$ \\
\hline Dual parents & $84 \cdot 5$ & 84.9 & $83 \cdot 8$ & $83 \cdot 5$ & $85 \cdot 4$ \\
\hline \multicolumn{6}{|l|}{ Sibling status } \\
\hline None & $30 \cdot 8$ & $33 \cdot 3$ & $27 \cdot 5$ & $29 \cdot 4$ & $32 \cdot 3$ \\
\hline One or more & $69 \cdot 2$ & $66 \cdot 7$ & $72 \cdot 5$ & $70 \cdot 6$ & $67 \cdot 7$ \\
\hline \multicolumn{6}{|l|}{ Brother status } \\
\hline None & $36 \cdot 3$ & $36 \cdot 6$ & $35 \cdot 9$ & $34 \cdot 1$ & $38 \cdot 6$ \\
\hline One or more & $63 \cdot 7$ & $63 \cdot 4$ & $64 \cdot 1$ & $65 \cdot 9$ & $61 \cdot 4$ \\
\hline \multicolumn{6}{|l|}{ Sister status } \\
\hline None & $42 \cdot 1$ & $36 \cdot 0$ & $50 \cdot 0$ & $40 \cdot 6$ & $43 \cdot 7$ \\
\hline One or more & $57 \cdot 9$ & $64 \cdot 0$ & $50 \cdot 0^{* *}$ & $59 \cdot 4$ & $56 \cdot 3$ \\
\hline
\end{tabular}

Younger adolescents, age range $12-15.5$ years, mean age 13.3 years; older adolescents, age range $14.6-16.5$ years, mean age $15 \cdot 6$ years. Values were significantly different from those of younger adolescents (Pearson's $\chi^{2}$ test of significance): ${ }^{\star \star} P<0 \cdot 01,{ }^{\star \star \star} P<0 \cdot 001$.

Table 2 Mean daily consumption of fruit, vegetables and unhealthy snacks, and mean weekly breakfast consumption, among younger and older adolescent boys and girls, East Midlands, UK, October 2007 to June 2008

\begin{tabular}{|c|c|c|c|c|c|c|c|c|}
\hline & \multicolumn{2}{|c|}{ Boys (n 186) } & \multicolumn{2}{|c|}{ Girls (n 142) } & \multicolumn{2}{|c|}{ Younger adolescents ( $n$ 170) } & \multicolumn{2}{|c|}{ Older adolescents ( $n$ 158) } \\
\hline & Mean & SD & Mean & SD & Mean & SD & Mean & SD \\
\hline Fruit consumption (portions/d) & $2 \cdot 5$ & $1 \cdot 7$ & $2 \cdot 5$ & $1 \cdot 6$ & $2 \cdot 4$ & $1 \cdot 7$ & $2 \cdot 7$ & $1 \cdot 7$ \\
\hline Vegetable consumption (portions/d) & $2 \cdot 1$ & $2 \cdot 1$ & $2 \cdot 2$ & $1 \cdot 5$ & 1.9 & $1 \cdot 7$ & $2 \cdot 5^{\star \star}$ & $2 \cdot 0$ \\
\hline Unhealthy snack consumption (times/d) & $3 \cdot 5$ & $3 \cdot 5$ & $2 \cdot 5^{\star \star}$ & $2 \cdot 5$ & $2 \cdot 9$ & $3 \cdot 1$ & $3 \cdot 2$ & $3 \cdot 4$ \\
\hline Breakfast consumption (d/week) & $5 \cdot 4$ & $2 \cdot 4$ & $4 \cdot 5^{\star *}$ & $2 \cdot 7$ & $5 \cdot 2$ & $2 \cdot 5$ & $4 \cdot 7$ & $2 \cdot 6$ \\
\hline
\end{tabular}

Mean values were significantly different from those of boys or younger adolescents (independent sample $t$ test): ${ }^{\star \star} P<0.01$.

the dietary behaviours assessed (Table 3). Significant main effects for family structure were only observed for breakfast consumption. Adolescents from dual-parent families $(P<0 \cdot 01)$ and adolescents with no brothers $(P<0.05)$ ate breakfast on more days per week than those from single-parent families and those with one or more brother, respectively.

Significant main effects for parenting style were observed for all dietary behaviours assessed except for vegetable consumption. For fruit consumption, across parental status $(P<0 \cdot 001)$, sibling status $(P<0 \cdot 001)$, brother status $(P<0 \cdot 01)$ and sister status $(P<0 \cdot 01)$ adolescents who described their parents as authoritative ate more fruit than those who described their parents as neglectful. In addition, across sibling $(P<0 \cdot 05)$, brother $(P<0.05)$ and sister status $(P<0.05)$ adolescents who described their parents as indulgent ate more fruit than those who described their parents as neglectful.

For unhealthy snack consumption, across parental status $(P<0 \cdot 05)$, sibling status $(P<0 \cdot 01)$, brother status $(P<0 \cdot 01)$ and sister status $(P<0 \cdot 01)$ adolescents who described their parents as authoritative ate fewer unhealthy snacks than those who described their parents as neglectful. In addition, across sibling status $(P<0 \cdot 05)$, brother status $(P<0.05)$ and sister status $(P<0.05)$ adolescents who described their parents as authoritarian ate fewer unhealthy snacks than those who described their parents as neglectful.

For breakfast consumption, across parental status $(P<0 \cdot 001)$, sibling status $(P<0 \cdot 001)$, brother status $(P<0 \cdot 001)$ and sister status $(P<0 \cdot 001)$ adolescents who described their parents as authoritative ate breakfast on more days per week than those who described their parents as neglectful. In addition, across sibling status $(P<0.05)$, brother status $(P<0.05)$ and sister status $(P<0 \cdot 05)$ adolescents who described their parents as authoritative ate breakfast on more days per week than those who described their parents as indulgent.

\section{Discussion}

The present study examined associations between parenting styles, family structure and adolescent dietary behaviours and the interaction of parenting styles and family structure variables with regard to adolescent dietary 
Table 3 Differences in adolescent dietary behaviours between the four parenting styles and family structure variables, East Midlands, UK, October 2007 to June 2008

\begin{tabular}{|c|c|c|c|c|c|c|c|c|c|c|c|c|}
\hline & \multicolumn{2}{|c|}{$\begin{array}{l}\text { Authoritative } \\
\text { (Av) (n 125) }\end{array}$} & \multicolumn{2}{|c|}{$\begin{array}{l}\text { Authoritarian } \\
\text { (At) (n 59) }\end{array}$} & \multicolumn{2}{|c|}{$\begin{array}{l}\text { Neglectful } \\
(\mathrm{Ng})(n 91)\end{array}$} & \multicolumn{2}{|c|}{$\begin{array}{l}\text { Indulgent } \\
\text { (In) }(n 53)\end{array}$} & \multirow{2}{*}{$\begin{array}{l}\text { Main effect: parenting } \\
\text { style ( } F \text { value })\end{array}$} & \multirow{2}{*}{$\begin{array}{l}\text { Main effect: family } \\
\text { structure ( } F \text { value })\end{array}$} & \multirow{2}{*}{$\begin{array}{l}\text { Interaction effect } \\
\quad(F \text { value })\end{array}$} & \multirow{2}{*}{$\begin{array}{c}\text { Post hoc tests: } \\
\text { Bonferroni contrasts }\end{array}$} \\
\hline & Mean & SD & Mean & SD & Mean & SD & Mean & SD & & & & \\
\hline \multicolumn{13}{|c|}{ Fruit consumption (portions/d) } \\
\hline \multicolumn{13}{|l|}{ Parental status } \\
\hline Single parent & $2 \cdot 98$ & 1.07 & $2 \cdot 61$ & 2.03 & 1.39 & $1 \cdot 21$ & $2 \cdot 45$ & $1 \cdot 66$ & & & & \\
\hline \multirow{2}{*}{\multicolumn{13}{|c|}{ Sibling status }} \\
\hline & & & & & & & & & & & & \\
\hline None & 3.06 & $2 \cdot 01$ & $1 \cdot 58$ & 1.45 & $1 \cdot 84$ & $1 \cdot 10$ & $2 \cdot 81$ & $1 \cdot 83$ & & & & \\
\hline One or more & $2 \cdot 60$ & $1 \cdot 64$ & $2 \cdot 77$ & $1 \cdot 67$ & $2 \cdot 12$ & $1 \cdot 66$ & $2 \cdot 86$ & $1 \cdot 60$ & $5 \cdot 87^{\star \star \star}$ & $1 \cdot 67$ & $2 \cdot 49$ & $\begin{array}{l}\mathrm{Av}>\mathrm{Ng}^{\star \star \star} \\
\ln >\mathrm{Ng}^{\star}\end{array}$ \\
\hline \multicolumn{13}{|l|}{ Brother status } \\
\hline None & $2 \cdot 96$ & 1.92 & $2 \cdot 67$ & $1 \cdot 39$ & $1 \cdot 82$ & $1 \cdot 13$ & $2 \cdot 68$ & $1 \cdot 82$ & & & & \\
\hline One or more & $2 \cdot 79$ & $1 \cdot 67$ & $2 \cdot 75$ & $1 \cdot 68$ & $2 \cdot 00$ & $1 \cdot 71$ & $2 \cdot 71$ & 1.59 & $5 \cdot 44^{\star \star \star}$ & $2 \cdot 46$ & $2 \cdot 12$ & $\begin{array}{l}\mathrm{Av}>\mathrm{Ng}^{\star *} \\
\ln >\mathrm{Ng}^{*}\end{array}$ \\
\hline \multicolumn{13}{|l|}{ Sister status } \\
\hline None & $2 \cdot 53$ & $1 \cdot 77$ & $2 \cdot 46$ & $1 \cdot 83$ & 1.98 & 1.59 & $2 \cdot 99$ & $1 \cdot 68$ & & & & \\
\hline One or more & $3 \cdot 16$ & $1 \cdot 73$ & $2 \cdot 54$ & $1 \cdot 62$ & $1 \cdot 88$ & $1 \cdot 47$ & $2 \cdot 49$ & $1 \cdot 66$ & $4 \cdot 23^{\star *}$ & $0 \cdot 42$ & $1 \cdot 69$ & $\begin{array}{l}\mathrm{Av}>\mathrm{Ng}^{\star *} \\
\ln >\mathrm{Ng}^{\star}\end{array}$ \\
\hline \multicolumn{13}{|c|}{ Vegetable consumption (portions/d) } \\
\hline \multicolumn{13}{|c|}{ Parental status } \\
\hline Single parent & $2 \cdot 04$ & $1 \cdot 14$ & 2.09 & 1.35 & $1 \cdot 21$ & 1.07 & $2 \cdot 34$ & $1 \cdot 52$ & & & & \\
\hline Dual parents & $2 \cdot 51$ & 1.94 & $1 \cdot 88$ & 1.64 & 1.94 & 1.89 & $2 \cdot 48$ & $2 \cdot 21$ & 1.35 & 0.85 & 0.61 & \\
\hline \multicolumn{13}{|l|}{ Sibling status } \\
\hline None & $2 \cdot 15$ & $1 \cdot 72$ & $1 \cdot 73$ & 1.62 & $2 \cdot 34$ & $2 \cdot 08$ & $3 \cdot 22$ & $2 \cdot 79$ & & & & \\
\hline One or more & $2 \cdot 39$ & 1.93 & 1.95 & 1.58 & $1 \cdot 71$ & 1.59 & $2 \cdot 28$ & $1 \cdot 71$ & $2 \cdot 31$ & $1 \cdot 68$ & $1 \cdot 87$ & \\
\hline \multicolumn{13}{|l|}{ Brother status } \\
\hline None & $2 \cdot 29$ & $1 \cdot 77$ & $1 \cdot 78$ & 1.55 & $2 \cdot 11$ & $2 \cdot 06$ & $2 \cdot 88$ & $2 \cdot 71$ & & & & \\
\hline One or more & 2.55 & 1.94 & 1.99 & 1.59 & 1.62 & 1.56 & $2 \cdot 18$ & $1 \cdot 73$ & 2.92 & 0.67 & $2 \cdot 40$ & \\
\hline \multicolumn{13}{|l|}{ Sister status } \\
\hline None & $2 \cdot 68$ & $2 \cdot 06$ & 1.69 & 1.08 & $1 \cdot 87$ & $1 \cdot 77$ & $2 \cdot 00$ & $1 \cdot 63$ & & & & \\
\hline One or more & $2 \cdot 25$ & $1 \cdot 66$ & 2.09 & 1.85 & $1 \cdot 73$ & $1 \cdot 79$ & $2 \cdot 83$ & $2 \cdot 44$ & $2 \cdot 79$ & 0.69 & 1.68 & \\
\hline \multicolumn{13}{|c|}{ Unhealthy snack consumption (times/d) } \\
\hline \multicolumn{13}{|c|}{ Parental status } \\
\hline Single parent & 1.91 & $1 \cdot 24$ & $2 \cdot 45$ & $1 \cdot 81$ & $3 \cdot 58$ & $4 \cdot 40$ & $1 \cdot 86$ & $1 \cdot 70$ & & & & \\
\hline \multirow{2}{*}{\multicolumn{13}{|c|}{ Sibling status }} \\
\hline & & & & & & & & & & & & \\
\hline None & $2 \cdot 34$ & 1.91 & $2 \cdot 28$ & 1.53 & $3 \cdot 71$ & 3.02 & $3 \cdot 33$ & $3 \cdot 51$ & & & & \\
\hline One or more & $2 \cdot 53$ & $2 \cdot 24$ & $2 \cdot 66$ & $2 \cdot 45$ & $4 \cdot 34$ & $5 \cdot 07$ & $2 \cdot 78$ & $2 \cdot 77$ & $3 \cdot 91^{* *}$ & 0.30 & 0.36 & $\begin{array}{l}\mathrm{Av}<\mathrm{Ng}^{\star \star} \\
\text { At }<\mathrm{Ng}^{*}\end{array}$ \\
\hline \multicolumn{13}{|l|}{ Brother status } \\
\hline None & $2 \cdot 52$ & $2 \cdot 39$ & $2 \cdot 53$ & $1 \cdot 56$ & 3.98 & $4 \cdot 65$ & $3 \cdot 33$ & $3 \cdot 48$ & & & & \\
\hline One or more & $2 \cdot 47$ & 1.99 & $2 \cdot 85$ & $2 \cdot 47$ & $4 \cdot 19$ & $4 \cdot 46$ & $2 \cdot 65$ & $2 \cdot 73$ & $4 \cdot 58^{* *}$ & 0.01 & 0.51 & $\begin{array}{l}\mathrm{Av}<\mathrm{Ng}^{* *} \\
\text { At }<\mathrm{Ng}^{*}\end{array}$ \\
\hline Sister status & & & & & & & & & & & & \\
\hline None & $2 \cdot 76$ & $2 \cdot 45$ & $2 \cdot 30$ & $2 \cdot 41$ & $3 \cdot 84$ & $5 \cdot 13$ & $2 \cdot 10$ & $1 \cdot 79$ & & & & \\
\hline One or more & $2 \cdot 28$ & $1 \cdot 82$ & $2 \cdot 88$ & $2 \cdot 14$ & $4 \cdot 26$ & $4 \cdot 18$ & $3 \cdot 41$ & $3 \cdot 63$ & $4 \cdot 24^{\star *}$ & $0 \cdot 82$ & $1 \cdot 27$ & $\begin{array}{l}\mathrm{Av}<\mathrm{Ng}^{\star \star} \\
\text { At }<\mathrm{Ng}^{\star}\end{array}$ \\
\hline
\end{tabular}




\begin{tabular}{|c|c|c|c|c|c|c|c|c|c|c|c|c|}
\hline & \multicolumn{2}{|c|}{$\begin{array}{l}\text { Authoritative } \\
(\mathrm{Av})(n \text { 125) }\end{array}$} & \multicolumn{2}{|c|}{$\begin{array}{l}\text { Authoritarian } \\
\text { (At) }(n 59)\end{array}$} & \multicolumn{2}{|c|}{$\begin{array}{l}\text { Neglectful } \\
(\mathrm{Ng})(n 91)\end{array}$} & \multicolumn{2}{|c|}{$\begin{array}{l}\text { Indulgent } \\
\text { (In) (n 53) }\end{array}$} & \multirow{2}{*}{$\begin{array}{l}\text { Main effect: parenting } \\
\text { style ( } F \text { value) }\end{array}$} & \multirow{2}{*}{$\begin{array}{l}\text { Main effect: family } \\
\text { structure }(F \text { value })\end{array}$} & \multirow{2}{*}{$\begin{array}{l}\text { Interaction effect } \\
\quad(F \text { value })\end{array}$} & \multirow{2}{*}{$\begin{array}{c}\text { Post hoc tests: } \\
\text { Bonferroni contrasts }\end{array}$} \\
\hline & Mean & SD & Mean & SD & Mean & SD & Mean & SD & & & & \\
\hline \multicolumn{13}{|c|}{ Breakfast consumption (d/week) } \\
\hline \multicolumn{13}{|l|}{ Parental status } \\
\hline Single parent & $4 \cdot 98$ & $2 \cdot 59$ & $3 \cdot 85$ & $3 \cdot 06$ & $2 \cdot 27$ & $2 \cdot 82$ & $4 \cdot 87$ & 3.32 & & & & \\
\hline Dual parents & $5 \cdot 82$ & $1 \cdot 91$ & $5 \cdot 25$ & $2 \cdot 54$ & $4 \cdot 66$ & $2 \cdot 72$ & $4 \cdot 93$ & $2 \cdot 50$ & $6 \cdot 87^{* * *}$ & $8 \cdot 09^{\star *}$ & $1 \cdot 26$ & $\begin{array}{l}\text { Av }>\mathrm{Ng}^{\star \star *} \\
\text { Dual }>\text { single }\end{array}$ \\
\hline \multicolumn{13}{|l|}{ Sibling status } \\
\hline None & $6 \cdot 15$ & 1.68 & $5 \cdot 80$ & $2 \cdot 24$ & $4 \cdot 51$ & 3.07 & $4 \cdot 83$ & $2 \cdot 23$ & & & & \\
\hline One or more & $5 \cdot 58$ & $2 \cdot 11$ & $4 \cdot 65$ & $2 \cdot 77$ & 3.95 & $2 \cdot 85$ & $4 \cdot 63$ & $2 \cdot 70$ & $6 \cdot 23^{* \star *}$ & $3 \cdot 23$ & $0 \cdot 19$ & $\begin{array}{l}\mathrm{Av}>\mathrm{Ng}^{\star * *} \\
\mathrm{Av}>\mathrm{In}^{*}\end{array}$ \\
\hline \multicolumn{13}{|l|}{ Brother status } \\
\hline None & $5 \cdot 97$ & $1 \cdot 84$ & $5 \cdot 61$ & $2 \cdot 29$ & $4 \cdot 58$ & $2 \cdot 91$ & $4 \cdot 88$ & $2 \cdot 22$ & & & & \\
\hline One or more & $5 \cdot 57$ & $2 \cdot 09$ & $4 \cdot 78$ & $2 \cdot 79$ & $3 \cdot 85$ & $2 \cdot 92$ & $4 \cdot 56$ & $2 \cdot 71$ & $6 \cdot 34^{\star \star *}$ & $3 \cdot 75^{\star}$ & $0 \cdot 21$ & $\begin{array}{l}\mathrm{Av}>\mathrm{Ng}^{* \star *} \\
\mathrm{Av}>\mathrm{In}^{\star} \\
\text { No brother }>\text { has } \\
\text { brothers }\end{array}$ \\
\hline \multicolumn{13}{|l|}{ Sister status } \\
\hline None & $5 \cdot 52$ & $2 \cdot 13$ & $5 \cdot 18$ & $2 \cdot 44$ & 4.33 & $2 \cdot 81$ & $4 \cdot 23$ & $2 \cdot 85$ & & & & \\
\hline One or more & $5 \cdot 85$ & $1 \cdot 89$ & $4 \cdot 91$ & $2 \cdot 85$ & $4 \cdot 08$ & $2 \cdot 99$ & $4 \cdot 99$ & $2 \cdot 25$ & $6 \cdot 07^{* * *}$ & 0.004 & 0.66 & $\begin{array}{l}\mathrm{Av}>\mathrm{Ng}^{\star \star \star *} \\
\mathrm{Av}>\mathrm{In}^{\star}\end{array}$ \\
\hline
\end{tabular}

Significance (analysis of covariance, analyses adjusted for gender and age of adolescent in the study): ${ }^{\star} P<0 \cdot 05,{ }^{\star \star} P<0 \cdot 01,{ }^{* \star \star} P<0 \cdot 001$. 
behaviours. Regardless of their family structure, adolescents who described their parents as authoritative had more favourable dietary behaviours than adolescents who described their parents as authoritarian, indulgent or neglectful.

A higher proportion of younger adolescents, compared with older adolescents, described their parents as authoritative and a higher proportion of older adolescents, compared with younger adolescents, described their parents as neglectful. Such findings support previous research $^{(35)}$ and suggest that approaches to intervene with parents and their adolescents should be age-specific because greater parental strictness and involvement is evident in earlier adolescence. Consistent with previous research, adolescent girls ate breakfast on fewer days per week compared with adolescent boys ${ }^{(30,36,37)}$. This may be a chosen method of weight control for girls, and in some individuals is associated with body dissatisfaction, dieting or disordered eating ${ }^{(36)}$. In the present study, adolescent boys ate more unhealthy snacks per day compared with girls. Furthermore, older adolescents ate more vegetables per day compared with younger adolescents. In contrast, review-level evidence has shown a negative association between age and fruit and vegetable consumption ${ }^{(38)}$. Contrasting findings may reflect a difference in the methodologies employed to assess fruit and vegetable consumption. Such differences in adolescent dietary behaviour suggest the importance of gaining an increased understanding of gender and age differences in specific dietary behaviours. Interventions aimed at promoting healthy dietary behaviours should target specific dietary behaviours by age and gender.

Adolescents who described their parents as authoritative ate more fruit, fewer unhealthy snacks per day and ate breakfast on more days per week than adolescents who described their parents as neglectful. Findings of the present study support previous research showing that adolescents raised in authoritative homes have higher fruit and vegetable consumption ${ }^{(24,25)}$ than adolescents raised in different types of household. An authoritative parenting style has also been positively associated with physical activity levels in girls ${ }^{(39)}$ and inversely associated with prevalence of overweight in children ${ }^{(40)}$. The association between an authoritative parenting style and positive dietary behaviours among adolescents may explain some of the contradictory findings in the literature on the influence of food-specific parenting practices, and in particular food rules, on adolescent dietary behaviours. General parenting style can be viewed as the emotional climate within which parenting practices occur. The emotional climate created by authoritative parents is one of high strictness and high involvement, responsiveness and warmth. It may be this combination of strictness, involvement and warmth in the interactions between parent and child that leads to positive dietary behaviours. Future research should assess the efficacy of strategies promoting the central attributes of an authoritative parenting style in an effort to support healthy dietary behaviours among young people. Drawing on tenets of self-determination theory, the encouragement and creation of autonomy-supportive environments could provide a useful starting point for parent education interventions $^{(41)}$.

There were additional differences between parenting styles that differed by food type. Consistent with previous research, adolescents who described their parents as indulgent ate more fruit per day than those who described their parents as neglectful ${ }^{(24)}$. Parental fruit consumption is positively associated with adolescent fruit consumption $^{(42)}$ and it may be that parents with attributes of an indulgent parenting style (warm and involved) are positive role models to their children without being strict about fruit consumption. On the other hand, adolescents who described their parents as authoritarian ate fewer unhealthy snacks per day than adolescents who described their parents as neglectful. Such findings support previous research where more food rules and more restrictiveness (attributes of an authoritarian parenting style) were related to less sweets, snacks and soft drinks $^{(19,20)}$. It may be the case that greater strictness is required to restrict unhealthy snack consumption, particularly when such snacks are likely to be highly available and reinforcing. However, long-term behaviour change may require a shift to more autonomy-driven behaviour of the adolescent. But this strategy has not been tested previously. For vegetable consumption, there were no significant main effects of parenting styles across any of the family structure variables. This could be related to how vegetables are available in households. It is expected that vegetable consumption is less regulated by the adolescent because servings will be provided by the parent as part of the meal. For breakfast consumption, adolescents who described their parents as authoritative ate breakfast on more days per week than those who described their parents as indulgent. Indulgent parents are warm and involved but not strict and may be more inclined to let their child decide on whether or not they want to eat breakfast than those parents who are strict and involved. Our findings offer support to previous research suggesting that influences vary by food type ${ }^{(43)}$, and reinforce the need for future research to be food-specific.

Consistent with findings from a recent systematic review, adolescents from dual-parent families ate breakfast on more days per week than those from single-parent families ${ }^{(29)}$. Such findings should be taken into consideration when designing programmes aimed at improving breakfast behaviours among adolescents. Also consistent with previous research, adolescents with no brothers ate breakfast on more days per week compared to those with one or more brother ${ }^{(30)}$. Such findings suggest the importance of gaining an increased understanding of the mechanisms underpinning associations 
between the presence of brothers and adolescent breakfast behaviours, as well as the mechanisms underpinning differences in breakfast behaviours of adolescents from single- and dual-parent families.

There were no significant interactions between parenting styles and family structure with adolescent dietary behaviours. The general absence of such interactions suggests that the benefit of authoritative parenting and the detriment of neglectful parenting transcend family structure for adolescent dietary behaviours. Such findings support previous research where, regardless of their ethnicity, class or parental marital status, adolescents with authoritative parents were more integrated at school, earned higher grades, were more self-reliant, reported less anxiety and depression, and were less likely to engage in delinquent behaviours, compared with those having neglectful parents ${ }^{(26,32,35)}$. Findings suggest that the most significant differences between families of differing structures may be in the resources available to facilitate healthy eating, rather than in the specific constellations of attitudes, values and beliefs.

Strengths of the present study include its high response rate and the analyses of individual dietary behaviours. Also the study is innovative in that it examined differences in adolescent dietary behaviours according to parenting styles and multiple indicators of family structure. However, findings should be viewed in light of the following limitations. Several studies have identified overestimation of fruit and vegetable intake when using $\mathrm{FFQ}^{(24,44)}$. Although the YAQ is probably the most suitable and well-tested ${ }^{(34,45)}$ tool for assessing dietary intake among adolescents, there are problems inherent to assessing dietary intake with self-reported measures. Given that the fruit and vegetable consumption levels reported by participants in the present study are higher than the national averages for England ${ }^{(46)}$, it may be that adolescents have over-reported because such behaviours are perceived as healthy and socially desirable ${ }^{(24)}$. The study is also limited by its cross-sectional design and the generalisability of the results is limited because participants were predominantly white and of upper socio-economic position, and so does not represent the population at large. Future research should investigate the associations between parenting styles, family structure and adolescent dietary behaviour in a sample with lower socio-economic position. The study used adolescent-reported general parenting style whereas future research may benefit from using aggregated information obtained from both parents and adolescents.

In summary, adolescents who described their parents as authoritative had more positive dietary behaviours than adolescents who described their parents as neglectful. The positive associations between authoritative parenting style and adolescent dietary behaviour transcend family structure. Future research should be food-specific and assess the efficacy of strategies promoting the central attributes of an authoritative parenting style on the dietary behaviours of adolescents from a variety of family structures.

\section{Acknowledgements}

Sources of funding: N.P. and A.J.A. are supported by a Research Studentship from Loughborough University. Conflict of interest declaration: No conflicts of interest. Authors' contributions: N.P. analysed the data and conceived and drafted the original manuscript. A.J.A., S.J.H.B., T.G. and C.E. provided critical feedback on all drafts. All authors read and approved the final manuscript. Acknowledgement: We thank Dr Rock Braithwaite, Humboldt State University, California for his help with data collection.

\section{References}

1. Currie C, Robert C, Morgan A et al. (2004) Young People's Health in Context. Health Behaviour in School-aged Children (HBSC): International Report from the 2001/ 2002 Survey. WHO Health Policy Series: Health Policy for Children and Adolescents no. 4. Copenhagen: WHO Regional Office for Europe.

2. Guenther PM, Dodd KW, Reedy J et al. (2006) Most Americans eat much less than recommended amounts of fruits and vegetables. J Am Diet Assoc 106, 1371-1379.

3. Story M \& Neumark-Sztainer D (1999) Promoting healthy eating and physical activity in adolescents. Arch Pediatr Adolesc Med 10, 109-123.

4. World Health Organization (2002) The World Health Report. Reducing Risks, Promoting Healthy Life. Geneva: WHO.

5. World Health Organization (2003) Diet, Nutrition and the Prevention of Chronic Diseases. Report of a Joint WHO/FAO Expert Consultation. Geneva: WHO.

6. Rennie KL, Johnson L \& Jebb SA (2005) Behavioural determinants of obesity. Best Pract Res Clin Endocrinol Metab 19, 343-358.

7. Crossman A, Anne Sullivan D \& Benin M (2006) The family environment and American adolescents' risk of obesity as young adults. Soc Sci Med 63, 2255-2267.

8. Mikkila V, Rasanen L, Raitakari OT et al. (2004) Longitudinal changes in diet from childhood into adulthood with respect to risk of cardiovascular diseases: the Cardiovascular Risk in Young Finns Study. Eur J Clin Nutr 58, 1038-1045.

9. Lien N, Lytle LA \& Klepp KI (2001) Stability in consumption of fruit, vegetables and sugary foods in a cohort from age 14-21. Prev Med 33, 217-226.

10. Tinsley BJ (2003) How Children Learn To Be Healthy. Cambridge: Cambridge University Press.

11. Newman K, Harrison L, Dashiff C et al. (2008) Relationships between parenting styles and risk behaviors in adolescent health: an integrative literature review. Rev Lat Am Enfermagem 16, 142-150.

12. Baumrind D (1991) Parenting styles and adolescent development. In The Encyclopedia of Adolescence, pp. 746-758 [J Brooks-Gunn, R Lerner and AC Petersen, editors]. New York: Garland.

13. Maccoby EE \& Martin JA (1983) Socialization in the context of the family: parent-child interaction. In Handbook of Child Psychology. vol. 4: Personality and Social Development, pp. 1-110 [EM Hetherington, editor]. New York: Wiley.

14. Baumrind D (1971) Current patterns of parental authority. Dev Psychol Monogr 4, 1 Part 2. 
15. Baumrind D (1967) Child care practices anteceding three patterns of preschool behavior. Genet Psychol Monogr $\mathbf{7 5}$, 43-88.

16. Darling N \& Steinberg L (1993) Parenting styles as a context: an integrative model. Psychol Bull 113, 487-496.

17. Birch LL \& Fisher JO (2000) Mothers' child-feeding practices influence daughters' eating and weight. $A m J$ Clin Nutr 71, 1054-1061.

18. Fisher JO \& Birch LL (1999) Restricting access to palatable foods affects children's behavioral response, food selection, and intake. Am J Clin Nutr 69, 1264-1272.

19. De Bourdeaudhuij I (1997) Family food rules and healthy eating in adolescents. J Health Psychol 2, 45-56.

20. van der Horst K, Kremers S, Ferreira I et al. (2007) Perceived parenting style and practices and the consumption of sugar-sweetened beverages by adolescents. Health Educ Res 22, 295-304.

21. De Bourdeaudhuij I, Te Velde SJ, Maes L et al. (2009) General parenting styles are not strongly associated with fruit and vegetable intake and social-environmental correlates among 11-year-old children in four countries in Europe. Public Health Nutr 12, 259-266.

22. Cullen KW, Baranowski T, Rittenberry L et al. (2000) Socioenvironmental influences on children's fruit, juice and vegetable consumption as reported by parents: reliability and validity of measures. Public Health Nutr 3, 345-356.

23. Young EM, Fors SW \& Hayes DM (2004) Associations between perceived parent behaviors and middle school student fruit and vegetable consumption. J Nutr Educ Behav 36, 2-8.

24. Kremers SP, Brug J, de Vries H et al. (2003) Parenting style and adolescent fruit consumption. Appetite 41, 43-50.

25. Lytle LA, Varnell S, Murray DM et al. (2003) Predicting adolescents' intake of fruits and vegetables. J Nutr Educ Behav 35, 170-175.

26. Steinberg L, Mounts NS, Lamborn SD et al. (1991) Authoritative parenting and adolescent adjustment across varied ecological niches. J Res Adolesc 1, 19-36.

27. Sessa FM \& Steinberg L (1991) Family structure and the development of autonomy during adolescence. J Early Adolesc 11, 38-55.

28. Berns RM (2006) Child, Family, School, Community: Socialization and Support, 7 th ed. Belmont, CA: Thomson Wadsworth.

29. Pearson N, Biddle SJH \& Gorely T (2009) Family correlates of breakfast consumption among children and adolescents: a systematic review. Appetite 52, 1-7.

30. Pearson N, MacFarlane A, Crawford D et al. (2009) Family circumstance and adolescent dietary behaviours. Appetite 52, 668-674.

31. Steinberg L, Elmen J \& Mounts N (1989) Authoritative parenting, psychosocial maturity, and academic success among adolescents. Child Dev 60, 1424-1436.

32. Lamborn SD, Mounts N, Steinberg L et al. (1991) Patterns of competence and adjustment among adolescents from authoritative, authoritarian, indulgent and neglectful families. Child Dev 62, 1049-1065.

33. Den Exter Blokland EAW, Engels RC \& Finkenauer C (2001) Parenting styles, self control and male juvenile delinquency: the mediating role of self-control. In Prevention and Control of Aggression and the Impacts on Its Victims, pp. 201-207 [M Martinez, editor]. Dordrecht/New York: Kluwer/Plenum Press.

34. Rockett HR, Breitenbach M, Frazier AL et al. (1997) Validation of a Youth/Adolescent Food Frequency Questionnaire. Prev Med 26, 808-816.

35. Shucksmith J, Hendry LB \& Glendinning A (1995) Models of parenting: implications for adolescent well-being within different types of family contexts. J Adolesc 18, 253-270.

36. Keski-Rahkonen A, Kaprio J, Rissanen A et al. (2003) Breakfast skipping and health-compromising behaviors in adolescents and adults. Eur J Clin Nutr 57, 842-853.

37. Rashidi A, Mohammadpour-Ahranjani B, Karandish M et al. (2007) Obese and female adolescents skip breakfast more than their non-obese and male peers. Cent Eur J Med 2, 481-487.

38. Rasmussen M, Krolner R, Klepp KI et al. (2006) Determinants of fruit and vegetable consumption among children and adolescents: a review of the literature. Part I: quantitative studies. Int J Behav Nutr Phys Act 3, 22.

39. Schmitz KH, Lytle LA, Phillips GA et al. (2002) Psychosocial correlates of physical activity and sedentary leisure habits in young adolescents: the Teens Eating for Energy and Nutrition at School study. Prev Med 34, 266-278.

40. Rhee KE, Lumeng JC, Appugliese DP et al. (2006) Parenting styles and overweight status in first grade. Pediatrics 117, 2047-2054.

41. Williams GC (2002) Improving patients' health through supporting the autonomy of patients and providers. In Handbook of Self-determination Research, pp. 233-254 [EL Deci and RM Ryan, editors]. Rochester, NY: The University of Rochester Press.

42. Pearson N, Biddle SJH \& Gorely T (2008) Family correlates of fruit and vegetable consumption in children and adolescents: a systematic review. Public Health Nutr 12, 267-283.

43. Baranowski T, Cullen KW \& Baranowski J (1999) Psychosocial correlates of dietary intake: advancing dietary intervention. Annu Rev Nutr 19, 17-40.

44. Van Assema P, Brug J, Ronda G et al. (2002) A short Dutch questionnaire to measure fruit and vegetable intake: relative validity among adults and adolescents. Nutr Health 16, 85-106.

45. Rockett HRH, Wolf AM \& Colditz GA (1995) Development and reproducibility of a food frequency questionnaire to assess diets of older children and adolescents. J Am Diet Assoc 95, 336-340.

46. The Information Centre for Health and Social Care (2006) Health Survey for England 2006. London: The Stationery Office. 